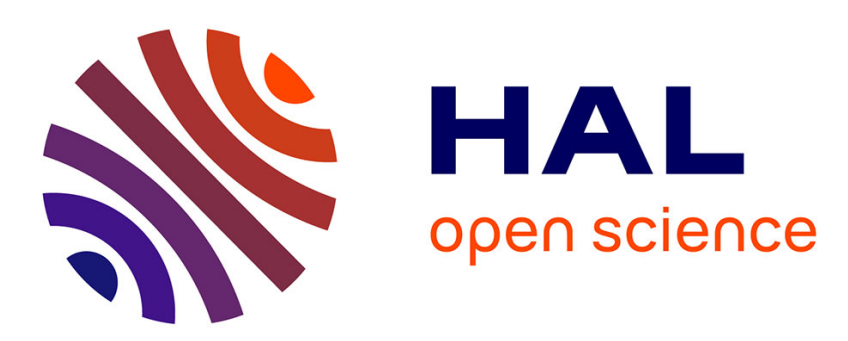

\title{
A characterization by optimization of the Monge point of a tetrahedron
}

Nicolas Hadjisavvas, Jean-Baptiste Hiriart-Urruty, Pierre-Jean Laurent

\section{To cite this version:}

Nicolas Hadjisavvas, Jean-Baptiste Hiriart-Urruty, Pierre-Jean Laurent. A characterization by optimization of the Monge point of a tetrahedron. Journal of Optimization Theory and Applications, 2016. hal-01975593

\section{HAL Id: hal-01975593 https://hal.science/hal-01975593}

Submitted on 9 Jan 2019

HAL is a multi-disciplinary open access archive for the deposit and dissemination of scientific research documents, whether they are published or not. The documents may come from teaching and research institutions in France or abroad, or from public or private research centers.
L'archive ouverte pluridisciplinaire HAL, est destinée au dépôt et à la diffusion de documents scientifiques de niveau recherche, publiés ou non, émanant des établissements d'enseignement et de recherche français ou étrangers, des laboratoires publics ou privés. 


\title{
A characterization by optimization of the MONGE point of a tetrahedron
}

\author{
Nicolas Hadjisavvas, Jean-Baptiste Hiriart-Urruty \& Pierre-Jean Laurent
}

\begin{abstract}
. "... nihil omnino in mundo contingint, in quo non maximi minimive ratio quapiam eluceat", translated into "... nothing in all the world will occur in which no maximum or minimum rule is somehow shining forth", used to say L.EulER in 1744. This is confirmed by numerous applications of mathematics in physics, mechanics, economy, etc. In this note, we show that it is also the case for the classical "centers" of a tetrahedron, more specifically for the so-called MONGE point (the substitute of the notion of orthocenter for a tetrahedron). To the best of our knowledge, the characterization of the Monge point of a tetrahedron by optimization, that we are going to present, is new.
\end{abstract}

\section{To begin with... What kind of tetrahedron?}

Let $\mathcal{T}=A B C D$ be a tetrahedron in the three dimensional space $\mathbb{R}^{3}$ (equipped with the usual Euclidean and affine structures); the points $A, B, C, D$ are supposed not to lie in a plane, of course. We begin with two particular types of tetrahedra and, then, with increase in generality, we can classify the tetrahedra into several classes. Here they are:

- The regular tetrahedron. This tetrahedron enjoys so many symmetries that it is not very interesting from the optimization viewpoint: all the "centers" usually associated with a tetrahedron (and that we are going to visit again in the next paragraph) coincide.

- The trirectangular tetrahedra. They are generalizations to the space of rectangular triangles in the plane. A trirectangular tetrahedron $O A B C$ has (two by two) three perpendicular faces $O B C, O A B, O A C$ and a "hypothenuse-face" $A B C$; such a tetrahedron enjoys a remarkable relationship between areas of its faces (see [1]); its vertex $O$, opposite the hypothenuse-face, is the orthocenter and Monge point, as we shall see below.

- The orthocentric tetrahedra. Curiously enough, the four altitudes of a tetrahedron generally do not meet at a point; when this happens, the tetrahedron is called orthocentric. A common characterization of orthocentric tetrahedra is as follows: a tetrahedron is orthocentric if and only if the opposite edges (two by two) are orthogonal. This class of tetrahedra is by far the most studied one in the literature. Regular and trirectangular tetrahedra are indeed orthocentric.

- General tetrahedra. Like for triangles, three specific "centers" can be defined for any tetrahedron: the centroid or isobarycenter, the incenter and the circumcenter. We shall see their characterization by optimization, as for some other points, in the next section. As said before, the altitudes do not necessarily meet at a point; moreover, the projection of any vertex on the opposite face does not necessarily coincide with the orthocenter of this face. The notion of orthocenter will be held by a new point: the so-called Monge point. 


\section{Characterization by optimization of the centroid, incenter, circumcenter, etc. of a tetrahedron}

Let us revisit the usual centers of a tetrahedron $\mathcal{T}=A B C D$ and their characterizations by optimization. They are similar to the ones developed for the centers of a triangle in [2].

- The centroid or isobarycenter (also called center of mass, center of gravity) $G$ of $\mathcal{T}$ is the best known of them: it can be defined as the barycenter of the four vertices $A, B, C, D$ with equal weights. In terms of optimization, it is the point which minimizes (on $\mathcal{T}$ or on $\mathbb{R}^{3}$ ) the following objective function or criterion

$$
P \longmapsto f_{1}(P)=(P A)^{2}+(P B)^{2}+(P C)^{2}+(P D)^{2} .
$$

Should $f_{1}$ represent a temperature function, the point $G$ would be the coolest point. Indeed, $f_{1}$ is a quadratic strictly convex function. According to the well-known necessary and sufficient condition for optimality in unconstrained convex minimization, the $G$ point is characterized by the vectorial relation $\overrightarrow{\nabla f_{1}}(G)=\overrightarrow{0}$, which can be written here as

$$
\overrightarrow{G A}+\overrightarrow{G B}+\overrightarrow{G C}+\overrightarrow{G D}=\overrightarrow{0}
$$

- The incenter $I$ of $\mathcal{T}$ is the center of the largest sphere included in $\mathcal{T}$; it is also the point (in $\mathcal{T}$ ) equidistant from the four faces of $\mathcal{T}$. In terms of optimization, it is the point which minimizes (on $\mathcal{T}$ or on $\mathbb{R}^{3}$ ) the following function

$$
P \longmapsto f_{2}(P)=\max \left(P A^{\prime}, P B^{\prime}, P C^{\prime}, P D^{\prime}\right),
$$

where $A^{\prime}, B^{\prime}, C^{\prime}, D^{\prime}$ denote the projections of $P$ on the faces of $\mathcal{T}\left(A^{\prime}\right.$ lies in the face opposite the vertex $A$, and so on). This new temperature function $f_{2}$ is again strictly convex, but nondifferentiable.

- The circumcenter $O$ is the center of the smallest sphere containing $\mathcal{T}$; it is also the point equidistant from the four vertices of $\mathcal{T}$. When it lies in the interior of $\mathcal{T}$, this point is the one which minimizes on $\mathcal{T}$ (as also on $\mathbb{R}^{3}$ ) the following function

$$
P \longmapsto f_{3}(P)=\max (P A, P B, P C, P D) .
$$

As for $f_{2}$, this function $f_{3}$ is strictly convex and nondifferentiable.

The above characterizations of the incenter and circumcenter in terms of optimization do not seem to be well-known. The difficulty there is that they involve a nonsmooth convex function (to be minimized), while the function to be minimized in (1) is convex and smooth. However, people involved in approximation theory are familiar with such kinds of minimization problems. We add three further interesting points to our list, even far less known however.

- The first Lemoine point of $\mathcal{T}$ is the one which minimizes on $\mathcal{T}$ the function

$$
P \longmapsto f_{4}(P)=\left(P A^{\prime}\right)^{2}+\left(P B^{\prime}\right)^{2}+\left(P C^{\prime}\right)^{2}+\left(P D^{\prime}\right)^{2},
$$

where $A^{\prime}, B^{\prime}, C^{\prime}, D^{\prime}$ denote, as above, the projections of $P$ on the faces of $\mathcal{T}$. See [3, page 79] for another characterization of this point. 
- The second Lemoine point of $\mathcal{T}$ is, this time, the one which minimizes on $\mathcal{T}$ the function

$$
P \longmapsto f_{5}(P)=\left(P P_{A B}\right)^{2}+\left(P P_{A C}\right)^{2}+\left(P P_{A D}\right)^{2}+\left(P P_{B C}\right)^{2}+\left(P P_{B D}\right)^{2}+\left(P P_{C D}\right)^{2}
$$

where $P_{A B}, P_{A C}, \ldots$ denote the projections of $P$ on the six edges of $\mathcal{T}$.

In the case of a triangle, there is only one LEMOINE point ([3, page 24$])$; for a tetrahedron, there are two.

- The FERMAT or FERMAT \& TORRICCELli point of $\mathcal{T}$ is the one which minimizes the function

$$
P \longmapsto f_{6}(P)=P A+P B+P C+P D .
$$

It is usually located inside the tetrahedron, but it could also be one of the vertices.

Again, the functions appearing in (5), (6) and (7) are convex, but the one in (7) is not differentiable at the points $A, B, C$ or $D$. So, characterizing the FERMAT point is different if a vertex of the tetrahedron is a candidate for optimality or not; however, subdifferential calculus and optimality conditions for convex functions, be they smooth or nonsmooth, cover all the cases $([4$, chapter D $])$.

\section{Properties of the centroid: an example of reasoning with convexity}

Here we illustrate how techniques from convex analysis allow us to derive easily some properties of "centers" in a tetrahedron; for this, we choose the particular case of the centroid. Let $x_{A}, \ldots, x_{D}$ denote the vectors of cartesian coordinates of the points $A, \ldots, D$ and $x_{G}$ that of the barycenter $G$ of $(A, \alpha),(B, \beta),(C, \gamma),(D, \delta)$. Here, the weights $\alpha, \ldots, \delta$ are those of a convex combination (i.e., positive and summing up to 1 ); for the centroid, these weights are exactly equal. We express $x_{G}$ as a convex combination of $x_{A}, \ldots, x_{D}$ :

$$
x_{G}=\alpha x_{A}+\beta x_{B}+\gamma x_{C}+\delta x_{D} .
$$

We now proceed like in the proofs of results in convex analysis (page 28 in [4] for example) and transform (8) into:

$$
x_{G}=(\alpha+\beta)\left(\frac{\alpha}{\alpha+\beta} x_{A}+\frac{\beta}{\alpha+\beta} x_{B}\right)+(\gamma+\delta)\left(\frac{\gamma}{\gamma+\delta} x_{C}+\frac{\delta}{\gamma+\delta} x_{D}\right) ;
$$

or

$$
x_{G}=(\alpha+\beta+\gamma)\left(\frac{\alpha}{\alpha+\beta+\gamma} x_{A}+\frac{\beta}{\alpha+\beta+\gamma} x_{B}+\frac{\gamma}{\alpha+\beta+\gamma} x_{C}\right)+\delta x_{D} .
$$

In (9): $\frac{\alpha}{\alpha+\beta} x_{A}+\frac{\beta}{\alpha+\beta} x_{B}=: x_{A B}$ lies on the line-segment $A B$ (it is the midpoint of $A B$ in the case of centroid), $\frac{\gamma}{\gamma+\delta} x_{C}+\frac{\delta}{\gamma+\delta} x_{D}=: x_{C D}$ lies on the line-segment $C D$; therefore, the $x_{G}$ appears as a new convex combination of $x_{A B}$ and $x_{C D}$ (it is again the mid-point in the case of centroid). In (10): $\frac{\alpha}{\alpha+\beta+\gamma} x_{A}+\frac{\beta}{\alpha+\beta+\gamma} x_{B}+\frac{\gamma}{\alpha+\beta+\gamma} x_{C}=: x_{A B C}$ lies on the triangle $A B C$ (it is its centroid in the case where $\alpha=\beta=\gamma=\delta=1 / 4$ ). In doing so, we have 
proved, without referring to any geometric argument (angles, distances, vector calculus) that:

- the centroid $x_{G}$ lies exactly in the middle of the line-segments joining midpoints of edges (there are three situations like this);

- the centroid $x_{G}$ is situated on the lines joining vertices to centroids of the opposite triangles (there are four situations like this), the corresponding barycentric weights being $3 / 4$ and $1 / 4$.

See Figure 1 for a graphical illustration.

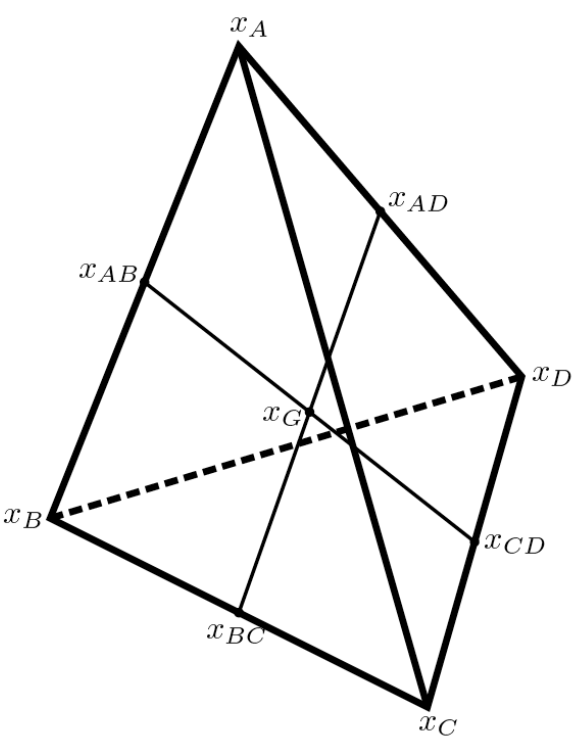

Joining midpoints of edges

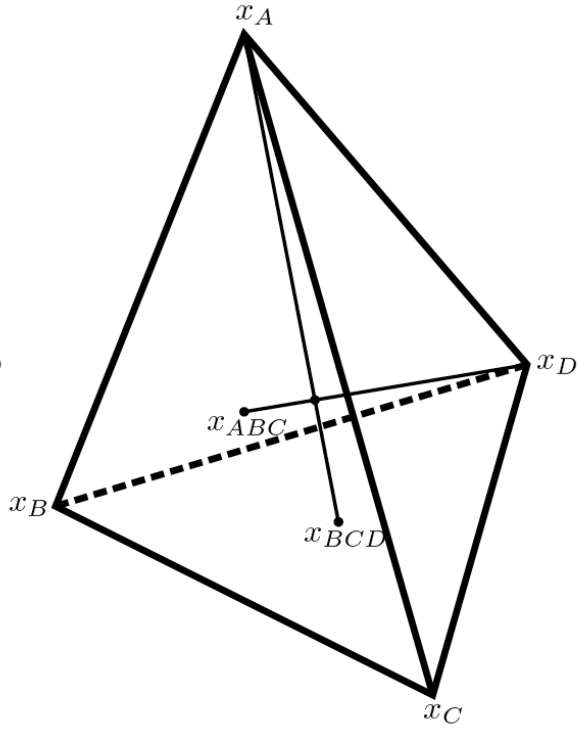

Joining vertices to centroids of triangles Figure 1

\section{The Monge point}

By an extraordinary geometrical intuition, G.Monge (1746-1818) proposed a substitute for the notion of orthocenter of a tetrahedron. Here is its basic definition. Consider the six planes perpendicular to the edges of a tetrahedron $\mathcal{T}$ and passing through the midpoints of the respective opposite sides; then these six planes meet at just one point; the point common to these planes is the so-called MONGE point of the tetrahedron. Our question is: can the MONGE point be viewed as the minimizer on $\mathcal{T}$ of some appropriate criterion? In other words, what is the temperature function on $\mathcal{T}$ (a convex, possibly differentiable, function) such that the coolest point is exactly the MoNGE point? Before answering this question, we review the main properties of the Monge point; they can be found in some classical books on solid geometry, or in the paper [5] that we recommend.

- If the tetrahedron is orthocentric, the orthocenter does exist as the intersection of the four altitudes, and the Monge point coincides with it. So, the Monge point is the direct generalization of the notion of orthocenter to arbitrary tetrahedra.

- If $G$ denotes the centroid of the tetrahedron and $O$ its circumcenter, the Monge point $M$ satisfies the following vectorial property: $\overrightarrow{O M}=2 \overrightarrow{O G}$. In other words, the MonGE point 
is the symmetric of the circumcenter with respect to the centroid.

- Another geometrical construction. A.MAnnheim's theorem (1895): the four planes determined by the four altitudes of a tetrahedron and the orthocenters of the corresponding faces pass through the MoNGE point. For example, in a trirectangular tetrahedron $O A B C$ (see section 1), the Monge point is the vertex $O$.

- The Monge point, more precisely its projection on faces, holds a compromise between the projections of vertices on faces and orthocenters of faces: the MoNGE point is equidistant from the orthocenter of a face and the projection on it of the opposite vertex (that holds true for the four faces). Again, in the case of orthocentric tetrahedra, the orthocenter of a face and the projection on it of the opposite vertex coincide; this common point is the projection of the orthocenter-MONGE point of the tetrahedron.

Now, we make the definition of MONGE point "variational", that is to say in terms of optimization. Our procedure will illustrate the following well-known adage: "This problem, when solved, will be simple". So, for each edge $\sigma$, denote by $\overrightarrow{v_{\sigma}}$ a unitary vector directing $\sigma$ (for example $\left(x_{B}-x_{A}\right) /\left\|x_{B}-x_{A}\right\|$ for the edge joining the vertices $A$ and $B$ ) and $x_{\sigma}$ the midpoint of the opposite edge ; then, since our aim is to look for a point $x$ such that $\left\langle x-x_{\sigma}, \overrightarrow{v_{\sigma}}\right\rangle=0$ for all $\sigma$, define the function

$$
P(\text { or vector } x) \longmapsto f_{7}(P)=\frac{1}{2} \sum_{\sigma_{1}}^{\sigma_{6}}\left[\left\langle x-x_{\sigma}, \overrightarrow{v_{\sigma}}\right\rangle\right]^{2},
$$

Theorem. The $f_{7}$ function is quadratic and stricly convex. It is uniquely minimized at the MONGE point.

Proof. As a sum of squares of affine forms, the $f_{7}$ function is clearly quadratic. The gradient vector $\nabla f_{7}(x)$ and the hessian matrix $\nabla^{2} f_{7}(x)$ of $f_{7}$ at $x$ are as follows:

$$
\begin{aligned}
\nabla f_{7}(x) & =\sum_{\sigma_{1}}^{\sigma_{6}}\left\langle x-x_{\sigma}, \overrightarrow{v_{\sigma}}\right\rangle \overrightarrow{v_{\sigma}} \\
\nabla^{2} f_{7}(x) & =\sum_{\sigma_{1}}^{\sigma_{6}} \overrightarrow{v_{\sigma}} \cdot\left(\overrightarrow{v_{\sigma}}\right)^{T} .
\end{aligned}
$$

To show that $\nabla^{2} f_{7}(x)$ is positive definite, consider the quadratic form $h \in \mathbb{R}^{3} \longmapsto$ $q(h)=\left\langle\nabla^{2} f_{7}(x) h, h\right\rangle=\sum_{\sigma_{1}}^{\sigma_{6}}\left\langle\overrightarrow{v_{\sigma}}, h\right\rangle^{2}$. Clearly, $q(h)$ is nonnegative on $\mathbb{R}^{3}$ and $q(h)=0$ if and only if $h=0$ (that is due to the fact that three among the six vectors $\overrightarrow{v_{\sigma}}$ are linearly independent). Hence the $f_{7}$ function is strictly convex.

The Monge point is indeed the unique minimizer of the nonnegative function $f_{7}$ since it is the only point where $f_{7}$ achieves the 0 value.

Remark 1. As the proof clearly shows, it suffices to consider in (11) a summation over three linearly independent edges, for example the three edges arising from a vertex. 
Remark 2. The $f_{7}$ function contains distances (to points or lines) in a hidden form. Indeed, consider the following construction: for each edge $\sigma$, let $\Delta_{\sigma}$ be the line passing through (the opposite midpoint) $x_{\sigma}$ and parallel to $\sigma$; we call it the mirror (edge or) line to $\sigma$. Then, because the vectors $\overrightarrow{v_{\sigma}}$ have been chosen unitary, the square of the distance from $x$ to the mirror line $\Delta_{\sigma}$ is

$$
d^{2}\left(x, \Delta_{\sigma}\right)=\left\|x-x_{\sigma}\right\|^{2}-\left[\left\langle x-x_{\sigma}, \overrightarrow{v_{\sigma}}\right\rangle\right]^{2} .
$$

So, the $f_{7}$ function is

$$
P(\text { or vector } x) \longmapsto f_{7}(P)=\frac{1}{2} \sum_{\sigma_{1}}^{\sigma_{6}}\left[\left\|x-x_{\sigma}\right\|^{2}-d^{2}\left(x, \Delta_{\sigma}\right)\right] .
$$

Other substitutes for the criterion to be minimized could be the following functions:

$$
\begin{gathered}
P(\text { or vector } x) \longmapsto f_{8}(P)=\sum_{\sigma_{1}}^{\sigma_{6}}\left[\left\|x-x_{\sigma}\right\|-d\left(x, \Delta_{\sigma}\right)\right] ; \\
P(\text { or vector } x) \longmapsto f_{9}(P)=\max _{\sigma_{1}, \ldots, \sigma_{6}}\left[\left\|x-x_{\sigma}\right\|-d\left(x, \Delta_{\sigma}\right)\right] .
\end{gathered}
$$

Hence, minimizing the $f_{8}$ function or the $f_{9}$ function yields again the MongE point. However, in comparison with the $f_{7}$ function, we loose properties like convexity and differentiability (on $\mathbb{R}^{3}$ ).

Remark 3. What about the numerical computation of the Monge point? We may use the (primary) definitions of this point as the intersection of appropriate planes. The results dealing with optimization, presented above, are also easily amenable to computations: either to solve the system $\nabla f_{7}(x)=0$ or, better, to minimize $f_{7}$ (summation over only three independent edges). In that case, the stopping rule of any minimization algorithm is: $\left|f_{7}\left(x_{k}\right)\right| \leqslant \epsilon$. In all cases, simple computations with MATLAB work well.

Remark 4. Back to the triangle ([6]). Analogous functions to those presented above can be defined to find (by minimization) the orthocenter of an acute triangle $A B C$. If $\sigma$ is one side of the triangle, $x_{\sigma}$ the vertex opposite to it, $\overrightarrow{v_{\sigma}}$ a unitary vector directing $\sigma$, and $\Delta_{\sigma}$ the line passing through $x_{\sigma}$ and parallel to $\sigma$, then the line orthogonal to $\sigma$ and passing through $x_{\sigma}$ has for equation: $\left\langle x-x_{\sigma}, \overrightarrow{v_{\sigma}}\right\rangle=0$, or $\left\|x-x_{\sigma}\right\|=d\left(x, \Delta_{\sigma}\right)$. As a result, the three functions $f_{7}, f_{8}, f_{9}$ (where the summations or the max operation are taken over the threes sides of the triangle) are minimized on $\mathbb{R}^{2}$ at a unique point: the orthocenter of the triangle. Actually, the $f_{8}$ function coincides on $\mathcal{T}$ with the one presented in [6], minus a constant (which equals the sum of the three altitudes of the triangle). This means, at least for an acute triangle: in order to find the orthocenter of a triangle, instead of minimizing the sum of distances from a point $P$ to the vertices, plus the sum of distances from $P$ to the sides, one could minimize the difference of two sums: the sum of the distances from $P$ to the vertices, and the sum of the distances from $P$ to the mirror sides (i.e., parallels to the sides, which are drawn through the vertices). That amounts to minimizing

$$
\begin{aligned}
P(\text { or vector } x) & \longmapsto \\
= & f_{10}(P)=P A+P B+P C-\left(d_{1}+d_{2}+d_{3}\right) \\
& (P A+P B+P C)+\left(P H_{1}+P H_{2}+P H_{3}\right)-\left(e_{1}+e_{2}+e_{3}\right),
\end{aligned}
$$


where the $e_{1}, e_{2}, e_{3}$ stand for the distances between the three sides and their mirrors (or the lengths of the three altitudes). See Figure 2.

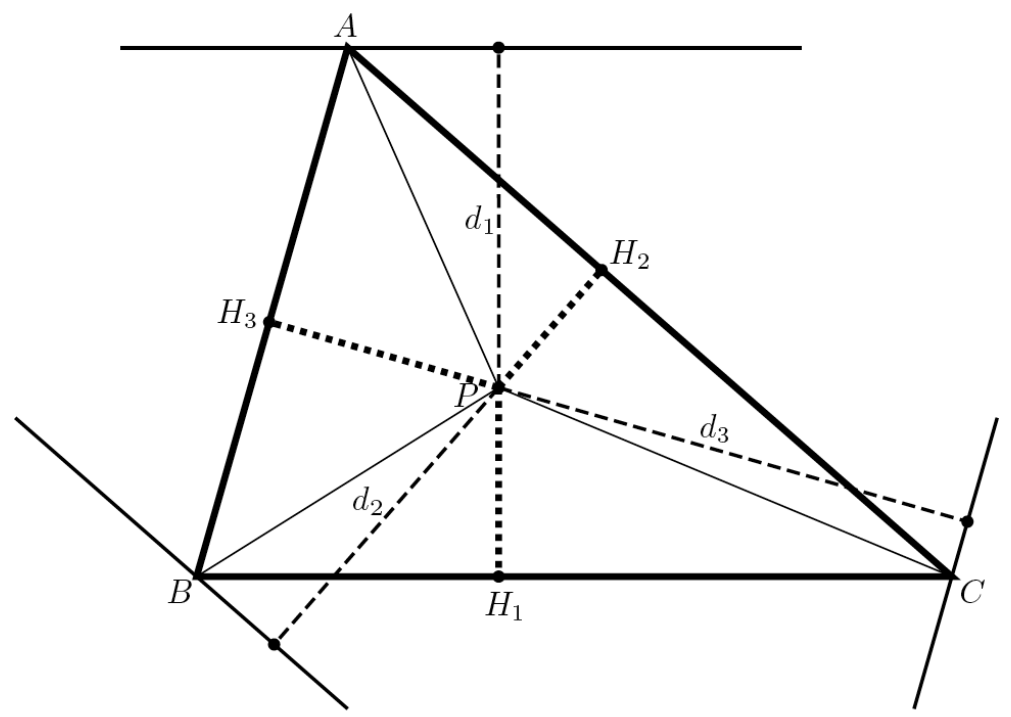

Figure 2: The case of a triangle

\section{Generalization to $\boldsymbol{n}$ dimensions}

Consider $n+1$ points in $\mathbb{R}^{n}$ that generate a convex polyhedron of full dimension $n$. The $\left(\begin{array}{c}n+1 \\ n\end{array}\right)$ hyperplanes that pass through the barycenters of any $n-1$ points and are orthogonal to the edge passing through the remaining two, meet exactly at one point. This point is called again the MoNGE point of the polyhedron; it has been studied recently in $[7,8]$. This point can also be found by minimizing a strictly convex function like in (11).

\section{References}

[1] J.-B.Hiriart-urruty, J.-P.Quadrat, J.B.Lasserre, Pythagoras' theorem for areas. American Math Monthly, Vol. 108, n6 (2001), $549-551$.

[2] J.-B.HIRIART-URRUTy, Quand la géométrie du triangle et l'optimisation convexe font bon ménage. To appear in Quadrature (2014).

[3] T.Andreescu, O.Mushkarov and L.Stoyanov, Geometric problems on maxima and minima. Birkhäuser (2006).

[4] J.-B.Hiriart-Urruty and C.Lemaréchal, Fundamentals of Convex Analysis. Grundlehren Text Editions. Springer (2001).

[5] R.A.Cross, Gaspard Monge and the Monge point of the tetrahedron. Mathematics Magazine, Vol. 76, n³ (2003), $193-203$.

[6] J.-B.Hiriart-URruty and P.-J.Laurent, A characterization of the orthocenter by optimization. To appear in Elemente der Mathematik (2014).

[7] M.BubA-Brozozowa, The Monge point and the $3(n+1)$ point sphere of an $n$ simplex. Journal for Geometry and Graphics, vol. 9, n¹, $31-36$ (2005). 
[8] M.HajJA and H.MARTini, Orthocentric simplices as the true generalizations of triangles. The Mathematical Intelligencer, vol. 35, n², $16-27$ (2013).

\section{The authors:}

Nicolas Hadjisavvas

Department of Product and Systems Design Engineering

University of the Aegean, Hermoupolis

84100 Syros, Greece.

www.syros.aegean.gr/users/nhad/

nhad@aegean.gr

JEAN-BAPTISTE HIRIART-URRUTY

Institut de Mathématiques

Université PAUL SABATIER

118, route de Narbonne

31062 Toulouse Cedex 9, France

www.math.univ-toulouse.fr/ jbhu/

jbhu@math.univ-toulouse.fr

Pierre-Jean Laurent

Laboratoire Jean Kuntzman (LJK)

Campus de Saint Martin d'Hères

Université Joseph Fourier, BP 53

38041 Grenoble Cedex 9, France

pjl@imag.fr 